\title{
Global analysis of HERA and RHIC data with a momentum space dipole model
}

\author{
E. A. F. Basso*, M. B. Gay Ducati* and E. G. de Oliveira ${ }^{\dagger}$ \\ *Instituto de Física, Universidade Federal do Rio Grande do Sul, Caixa Postal 15051, 91501-970, \\ Porto Alegre, RS, Brazil \\ ${ }^{\dagger}$ Instituto de Física, Universidade de São Paulo, Caixa Postal 66318, 05314-970, São Paulo, SP, \\ Brazil
}

\begin{abstract}
Within the $k_{t}$-factorization formalism we show the results on the charged hadron yield for $p+p$ collisions at LHC using the AGBS momentum space dipole scattering amplitude. This model is based on a correspondence between the LO Balitsky-Kovchegov evolution equation and the reaction-diffusion physics and was globally fitted to the DIS HERA data and inclusive hadron production on $p+p$ and $d+A u$ collisions at RHIC. Despite the fact it is a LO calculation, the present LHC data is quite well described.
\end{abstract}

Keywords: Inclusive hadron production, Nonlinear Evolution, Dipole Formalism

PACS: $12.38 . \mathrm{Bx}, 13.60 . \mathrm{Hb}, 13.85 . \mathrm{Ni}$

\section{THE DIPOLE AMPLITUDE IN MOMENTUM SPACE}

Among the various studies with the dipole amplitudes, there are very few using a momentum space parametrization. Here we will use the AGBS model proposed by de Santana Amaral, Gay Ducati, Betemps, and Soyez (AGBS) [1]. This model analytically interpolates between the behavior of the BK scattering amplitude in the dilute regime and the saturation one, in which it behaves like

$$
N(k, Y) \stackrel{k \ll Q_{s}}{=} c-\log \left(\frac{k}{Q_{s}(Y)}\right) .
$$

The expression for the tail of the scattering amplitude reads

$$
N(k, Y) \stackrel{k \gg Q_{s}}{\approx}\left(\frac{k^{2}}{Q_{s}^{2}(Y)}\right)^{-\gamma_{c}} \log \left(\frac{k^{2}}{Q_{s}^{2}(Y)}\right) \exp \left[-\frac{\log ^{2}\left(k^{2} / Q_{s}^{2}(Y)\right)}{2 \bar{\alpha} \chi^{\prime \prime}\left(\gamma_{c}\right) Y}\right] .
$$

Interpolation in the AGBS model is done through the following expression for the scattering amplitude $\left(\rho \equiv \ln \left(k^{2} / k_{0}^{2}\right)\right)$

$$
\tilde{T}^{\mathrm{AGBS}}(\rho, Y)=L_{F}\left(1-e^{-T_{\mathrm{dil}}}\right),
$$

where

$$
\begin{aligned}
& T_{\text {dil }}=\exp \left[-\gamma_{c}\left(\rho-\rho_{s}\right)-\frac{\mathscr{L}^{2}-\log ^{2}(2)}{2 \bar{\alpha} \chi^{\prime \prime}\left(\gamma_{c}\right) Y}\right], \\
& \text { Diffraction 2012 } \\
& \text { AIP Conf. Proc. 1523, 339-342 (2013); doi: 10.1063/1.4802181 } \\
& \quad \text { ( ) 2013 AIP Publishing LLC 978-0-7354-1146-3/\$30.00 }
\end{aligned}
$$



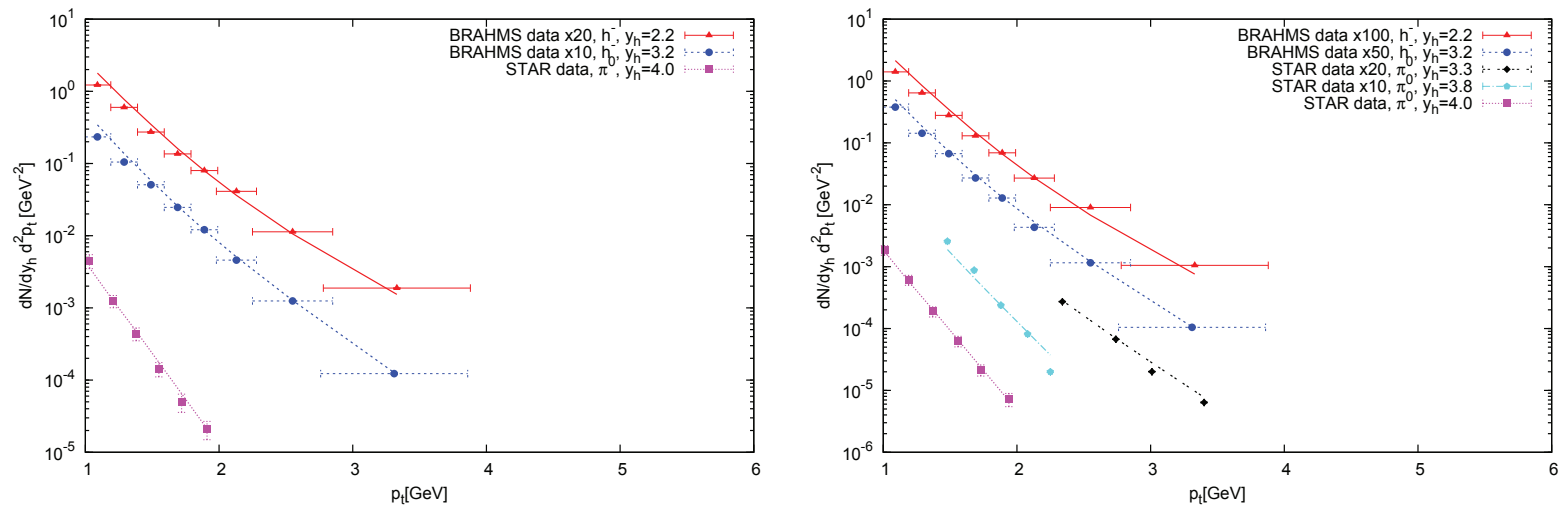

FIGURE 1. Results from the fit of the AGBS model for the RHIC charged hadron yield for $p+p$ (right) and $d+A u$ (left) collisions. Data taken from [5].

$$
\mathscr{L}=\ln \left[1+e^{\left(\rho-\rho_{s}\right)}\right] \quad \text { with } \quad Q_{S}^{2}(Y)=k_{0}^{2} e^{\lambda Y},
$$

and

$$
L_{F}=1+\ln \left[e^{\frac{1}{2}\left(\rho-\rho_{s}\right)}+e^{-\frac{1}{2}\left(\rho-\rho_{s}\right)}\right] .
$$

Such model was originally fitted to the proton structure function $F_{2}^{p}$, with good results even with the inclusion of heavy quarks. It was also used to investigate the possibility of gluon number fluctuations on the high energy evolution at HERA energies [2]. Recently it has been shown that the AGBS model describes equally well the DIS and inclusive hadron production in $p+A$ and $p+p$ collisions [3] within a hybrid formulation for the production cross section, where the projectile wave function is described with the DGLAP evolved PDFs while the target is considered as a dense system of gluons. This formulation is suited to describe the parton fragmentation region of the collision, that for increasing energy happens for more forward rapidities.

This study employed a new simultaneous fit to the HERA data on the proton structure function [4] and to the RHIC data on the $p_{t}$ distribution of the produced hadrons and pions from BRAHMS and STAR collaborations [5], for which the results are shown in the Figure 1. The model agrees with both data sets, where we used an educated ansatz for the nuclear geometry, for which the nuclear saturation scale reads $Q_{s, A}^{2}=A_{\mathrm{eff}}^{1 / 3} Q_{s, p}^{2}$, where $A_{\text {eff }}=18.5$ for gold nucleus. We know that such prescription does not cover the full density of partons over the transverse space of the colliding hadrons, but one can make an analysis of the presence or not of the saturation physics at the LHC.

\section{THE HADRON PRODUCTION WITH $k_{t}$-FACTORIZATION}

At very high energies and when the scattering is between a dilute projectile and a dense target, the main contribution is the gluon fusion, and the cross section can be written in a $k_{t}$-factorized form [6] 


$$
\frac{d \sigma^{A+B \rightarrow g}}{d y d^{2} p_{t}}=K \frac{2}{C_{F} p_{t}^{2}} \int^{p_{t}} \frac{d^{2} k_{t}}{4} \alpha_{S}(Q) \varphi\left(\frac{\left|p_{t}+k_{t}\right|}{2}, x_{1}\right) \varphi\left(\frac{\left|p_{t}-k_{t}\right|}{2}, x_{2}\right),
$$

where $x_{1,2}=\left(p_{t} / \sqrt{s}\right) e^{ \pm y}$ are momentum fractions of the incoming gluons and $C_{F}=$ $\left(N_{c}^{2}-1\right) / 2 N_{c}$ is the Casimir for the fundamental representation.

In the large $N_{c}$ limit, the unintegrated gluon distribution in either of the two colliding hadrons can be related to the dipole scattering amplitude through

$$
\varphi(k, x)=\frac{N_{c}}{2 \pi^{2} \alpha_{S}(k)} k^{2} \nabla_{k}^{2} N_{G}(k, x) .
$$

The charged hadron yield, given in terms of (7), reads

$$
\frac{d N_{c h}}{d \eta d^{2} p_{t}}=\frac{h[\eta]}{\sigma_{n s d}} \int d^{2} R \int \frac{d z}{z^{2}} D_{h}\left(z=p_{t} / k_{t}, \mu\right) \frac{d \sigma^{A+B \rightarrow g}}{d y d^{2} p_{t} d^{2} R},
$$

where $D_{h}\left(z=p_{t} / k_{t}, \mu\right)$ stands for the fragmentation function of the produced gluon into hadrons, for which we used the LO KKP model [7] with the scale $\mu=p_{t}$ of the hadron, $\sigma_{n s d}$ is the non-single-diffractive cross section taken from the KMR model [8], and $h[\eta]$ denotes the Jacobian for the transformation of the measured pseudorapidity into the rapidity entering the scattering amplitude. The $\alpha_{S}$ was allowed to vary within the LO prescription for $N_{f}=3$ light quark flavors

$$
\alpha_{S}\left(Q^{2}\right)=\frac{12 \pi}{27 \log \frac{Q^{2}}{\Lambda_{\mathrm{QCD}}^{2}}}
$$

with the maximum value of momentum of the UGDs as scale, $i$. e., we use $Q=$ $\max \left\{\left|p_{t}+k_{t}\right| / 2,\left|p_{t}-k_{t}\right| / 2\right\}$, and set $\Lambda_{\mathrm{OCD}}^{2}=0.05$.

The model describes quite well the LHC data [9] on both the high and small $p_{t}$ regions, as seen in Figure 2. Within the $k_{t}$-factorization formalism the $K$ factors become smaller than in the Hybrid one, as expected since in the former both colliding hadrons are treated symmetrically. Thus, the central rapidity region present in the averaged data for $|\eta|<2.4$ of the CMS collaboration introduces less errors into the $K$. In the $p-A$ case we note that the AGBS model works in the proton fragmentation region, where the small $x_{2}$ of the dense nucleus is been probed. In the negative rapidity region, one sees that the model still needs to take care of the large nuclear $x_{2}$ effects, such as the EMC and Fermi motion. As mentioned before, a full description of the nuclear geometry, with the inclusion of an impact parameter dependence in the model might help to cover all the nuclear effects one could not access in this work.

\section{ACKNOWLEDGMENTS}

This work is supported by CNPq (Brazil). E.G.O. is supported by FAPESP under contract 2011/50597-8. 

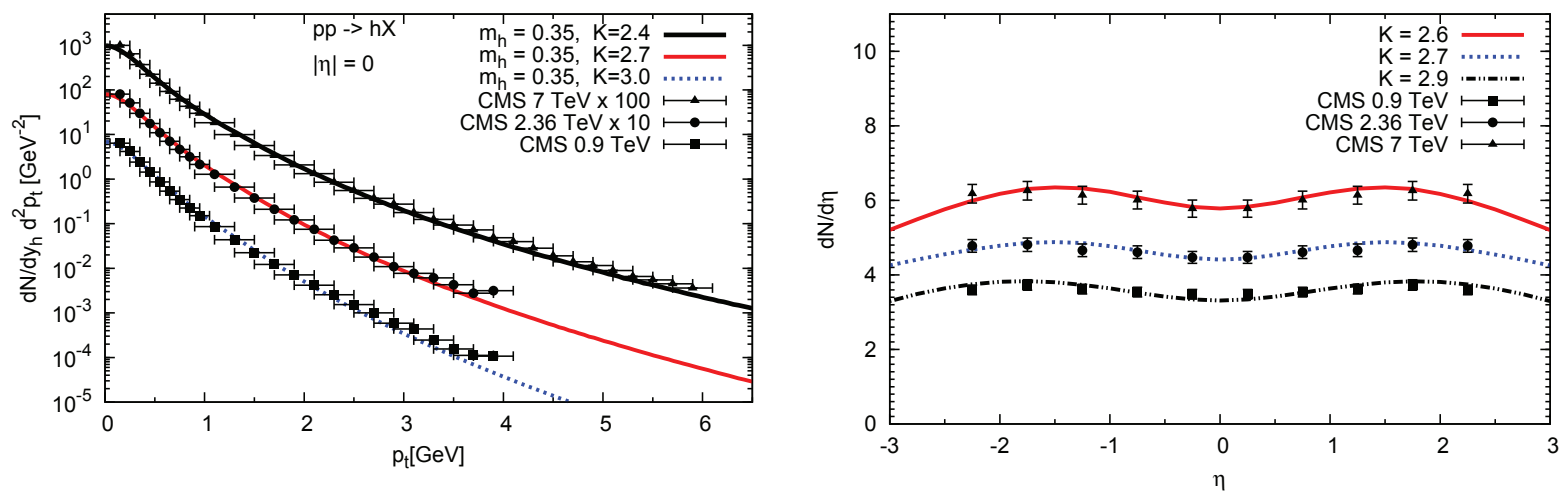

FIGURE 2. Predictions of the AGBS model for the LHC CMS charged hadron transverse momentum (left) and pseudorapidity (right) distributions for $p+p$ collisions at $\sqrt{s}=0.9,2.36$ and $7 \mathrm{TeV}$ [9].

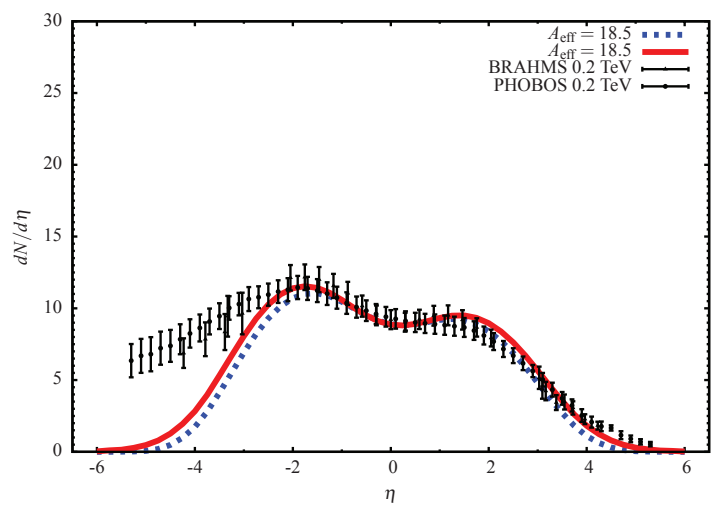

FIGURE 3. Predictions of the AGBS model to the RHIC pseudorapidity distribution for $d+A u$ collisions at $\sqrt{s}=200 \mathrm{GeV}[10]$.

\section{REFERENCES}

1. J. T. de Santana Amaral, M. B. Gay Ducati, M. A. Betemps and G. Soyez, Phys. Rev. D 76, 094018 (2007)

2. E. Basso, M. B. Gay Ducati, E. G. de Oliveira, and J. T. de Santana Amaral, Eur. Phys. J. C 58, 9 (2008); E. Basso, M. B. Gay Ducati, E. G. de Oliveira, and J. T. de Santana Amaral, Braz. J. Phys. v. 38, 483 (2008).

3. E. A. F. Basso, M. B. Gay Ducati and E. G. de Oliveira, Phys. Rev. D 84, 034024 (2011).

4. F. D. Aaron et al. (H1 and ZEUS Collaborations), JHEP 0110, 109 (2010).

5. I. Arsene et al. (BRAHMS Collaboration), Phys. Rev. Lett. 93, 242303 (2004); J. Adams et al. (STAR collaboration), Phys. Rev. Lett. 97, 152302 (2006).

6. M. Braun, Phys. Lett. B 483, 105 (2000); Y. V. Kovchegov and K. Tuchin, Phys. Rev. D 65, 074026 (2002).

7. B. A. Kniehl, G. Krammer and B. Pötter, Nucl. Phys. B582, 514 (2000).

8. M. G. Ryskin, A. D. Martin and V. A. Khoze, Eur. Phys. J. C 71, 1617 (2011).

9. V. Khachatryan et al. (CMS Collaboration), JHEP 0210, 041 (2010); Phys. Rev. Lett. 105, 022002 (2010).

10. I. Arsene et al. (BRAHMS Collaboration), Phys. Rev. Lett. 94, 0323011 (2005); B. B. Back et al. (PHOBOS Collaboration), Phys. Rev. Lett. 93, 082301 (2004). 
Copyright of AIP Conference Proceedings is the property of American Institute of Physics and its content may not be copied or emailed to multiple sites or posted to a listserv without the copyright holder's express written permission. However, users may print, download, or email articles for individual use. 